This is an electronic reprint of the original article. This reprint may differ from the original in pagination and typographic detail.

\author{
Author(s): Singkarat, S.; Puttaraksa, Nitipon; Unai, S.; Yu, L.D.; Singkarat, K.; Pussadee, N.; \\ Whitlow, H.J.; Natyanum, S.; Tippawan, U.
}

Title: Development of economic MeV-ion microbeam technology at Chiang Mai University

Year: $\quad 2017$

Version:

Please cite the original version:

Singkarat, S., Puttaraksa, N., Unai, S., Yu, L.D., Singkarat, K., Pussadee, N., Whitlow, H.J., Natyanum, S., \& Tippawan, U. (2017). Development of economic MeV-ion microbeam technology at Chiang Mai University. Nuclear Instruments and Methods in Physics Research Section B: Beam Interactions with Materials and Atoms, 404, 5864. https://doi.org/10.1016/j.nimb.2017.01.048

All material supplied via JYX is protected by copyright and other intellectual property rights, and duplication or sale of all or part of any of the repository collections is not permitted, except that material may be duplicated by you for your research use or educational purposes in electronic or print form. You must obtain permission for any other use. Electronic or print copies may not be offered, whether for sale or otherwise to anyone who is not an authorised user. 


\title{
Development of economic MeV-ion microbeam technology at Chiang Mai University
}

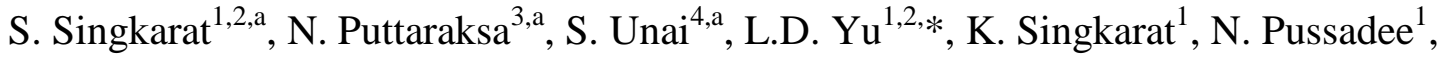 \\ H.J. Whitlow ${ }^{5}$, S. Natyanum ${ }^{1}$, and U. Tippawan ${ }^{1}$ \\ ${ }^{1}$ Plasma and Beam Physics Research Facility, Department of Physics and Materials Science, \\ Faculty of Science, Chiang Mai University, Chiang Mai 50200, Thailand \\ ${ }^{2}$ Thailand Center of Excellence in Physics, Commission on Higher Education, \\ 328 Si Ayutthaya Road, Bangkok 10400, Thailand \\ ${ }^{3}$ Nanoscience Center, P.O. Box 35, FI-40014 University of Jyväskylä, Finland \\ ${ }^{4}$ Physics Division, School of Science, University of Phayao, Phayao 56000, Thailand \\ ${ }^{5}$ Louisiana Accelerator Center, Department of Physics, University of Louisiana at Lafayette, \\ Lafayette, LA 70503-2067, USA
}

\begin{abstract}
Developing high technologies but in economic manners is necessary and also feasible for developing countries. At Chiang Mai University, Thailand, we have developed MeV-ion microbeam technology based on a 1.7-MV Tandetron tandem accelerator with our limited resources in a cost-effective manner. Instead of using expensive and technically complex electrostatic or magnetic quadrupole focusing lens systems, we have developed cheap MeV-ion microbeams using programmed L-shaped blade aperture and capillary techniques for MeV ion beam lithography or writing and mapping. The programmed L-shaped blade micro-aperture
\end{abstract}


system consists of a pair of L-shaped movable aperture pieces which are controlled by computer to cut off the ion beam for controlling the beam size down to the micrometer order. The capillary technique utilizes our home-fabricated tapered glass capillaries to realize microbeams. Either system can be installed inside the endstation of the $\mathrm{MeV}$ ion beam line of the accelerator. Both systems have been applied to $\mathrm{MeV}$-ion beam lithography or writing of micro-patterns for microfluidics applications to fabricate lab-on-chip devices. The capillary technique is being developed for $\mathrm{MeV}$-ion beam mapping of biological samples. The paper reports details of the techniques and introduces some applications.

Keywords: MeV ion; Microbeam; L-shaped blade aperture; tapered glass capillary; Lithography

*: Corresponding author: Email: yuld@thep-center.org

${ }^{a}$ : Authors contributed equally to the work.

\section{Introduction}

The ion microbeam technology is an extension and also a promotion of general ion beam technology. An ion microbeam is an ion beam of micrometer or sub-micrometer in the beam diameter. Together with integrated imaging techniques, microbeams allow precisely defined locations on the sample to be irradiated. The main applications of microbeam are in two aspects: (1) processing materials - highly localized and highly controlled ion quantity irradiation of the sample, and (2) analyzing materials - imaging/mapping and probing of the sample. Microbeam at $\mathrm{MeV}$ energy levels is more advantageous than lower energy microbeam in applying ion beam 
analysis techniques such as particle-induced X-ray emission (PIXE) for mapping analysis of samples and $\mathrm{MeV}$-ion irradiation of materials for more effective modification or fabrication. Since the first microbeam facilities were developed in the mid-1990s [1], nowadays, there are about 50 microbeam facilities all over the world [2].

Beam spots with a diameter down to about two micrometers can be obtained by collimating the beam with pinhole apertures or with a drawn capillary. Sub-micrometer beam spot sizes can be achieved by focusing the beam using various combinations of electrostatic or magnetic lenses. Both methods are used at present. Certainly the method of using focusing devices is considerably expensive compared to the method of using beam confining devices. For example, the cost of a triplet quadrupole magnetic focusing lens system for $\mathrm{MeV}$-ion beams, although highly variable, can be up to million US dollars, while that of a beam collimation and aperture system for the same energy ion beams may only be about a hundredth of the former. Moreover, utilization of such a system requires high stability of a power supply system. Based on our limited resources and locally practical power supply quality, we have adopted the more economic ways to establish the $\mathrm{MeV}$ microbeam technology at Chiang Mai University (CMU) with some technical developments in adapting such microbeams to the micrometersubmicrometer levels for applications in $\mathrm{MeV}$-ion-beam lithography (at present) as well as imaging/mapping (under development). Two techniques have been developed or adopted, namely programmable proximity aperture and tapered glass capillary. Utilization of the programmable proximity aperture for $\mathrm{MeV}$ ion beam lithography was initially developed by the groups in both University of Jyvaskyla and CMU [3]. Although the technique does not have a comparable resolution with that of using the focused proton beam lithography technique [4], it has the significant advantage of fast writing especially for large areas [5]. The use of tapered 
glass capillary for focusing $\mathrm{MeV}$ light ion beam has been developed in a number of studies (e.g. [6-8]). As a novel and new way to focus ion beams, some theories involved such as ion transmission and focusing mechanism and effect have been investigated. The technique has been demonstrated to be cost-effective in obtaining focused ion beams for various applications though the beam size currently remains yet at micrometer in minimum. A noticeable difference between the aperture and the capillary techniques is in the focusing effect which is described by the focusing factor $(f)$ as

$f=($ Outlet beam current density)/(Inlet beam current density).

For the aperture method, always $f=1$, while for the capillary method, $f>1$ [9].

\section{Facilities}

The CMU MeV microbeam facility is based on our 1.7-MV Tandetron tandem accelerator (High Voltage Engineering Europa, or HVEE) and its beam line. As shown in Fig. 1, the entire accelerator and beam line (donated by the Chalmers University of Technology in Gothenburg, Sweden) consist of an ion source terminal which includes two ion sources, a duoplasmatron ion source (Peabody Scientific) and a Cs sputter ion source (Peabody Scientific), a switching magnet with two entrances and one exit, a low-energy ion implantation chamber, the accelerator including the high-voltage supply, the post-acceleration beam transportation line including a $30^{\circ}$ mass analyzing magnet and quadrupole lenses, and an ion-beam analysis and lithography chamber. The duoplasmatron ion source allows the extraction of alpha particles and protons and the negative ion sputter source is capable of producing heavy negative ion species. The ions extracted from the ion source by $30 \mathrm{kV}$ can be applied for ion implantation at the small 
target chamber right after the switching magnet. The tandem accelerator has a terminal voltage up to $1.7 \mathrm{MV}$. At the terminal electrode, a nitrogen gas stripper converts negative ions to positive ones and allows a second acceleration of the ions with the same voltage. The accelerated ions are transported in the beam line through focusing lenses and the mass analyzing magnet to enter the main target chamber. The end-station chamber, which has been greatly upgraded by ourselves from the original design when it was used in Sweden, has two functions, i.e. ion beam analysis and lithography. For ion beam analysis, the chamber contains a sample stage on a 2-D translatable and 6-axis rotatable goniometer, and detectors such as SSB (silicon surface barrier) detector, $\mathrm{Si}(\mathrm{Li})$ detector and optical fibers for Rutherford Backscattering Spectrometry (RBS), Elastic non-Rutherford Backscattering (EBS), Particle Induced X-ray Emission (PIXE) and Ionoluminescence (IL) analyses, respectively. For microbeam lithography, systems of a programmable L-shape micro-aperture [10] and a tapered glass micro-capillary [11] have been developed and installed in the chamber, respectively.

The micro-aperture system, as shown in Fig. 2, is composed of a pair of L-shaped aperture blades, an ion beam fluence monitor and a beam shutter. The key component is the pair of L-shaped aperture blades which were made from tantalum with well-polished edges. The blades are computerized for smooth translation. The combination of the movements of the pair of the blades in $\mathrm{X}$ - and $\mathrm{Y}$-directions realizes a micro-aperture which controls the microbeam in a micrometer rectangular or square shape with a size down to $1 \mu \mathrm{m}$ in minimum. The two blades are mounted onto MM-3M-F-1 Motorized MicroMini ${ }^{\mathrm{TM}}$ stages with $0.49609 \mu \mathrm{m} / \mathrm{step}$ and 25.4 $\mathrm{mm}$ travel range. One of the stages can move in the $\mathrm{X}$ direction and the other in the $\mathrm{Y}$ direction. The sample is mounted on an X-Y stage of the goniometer. Cooperating with X-Y translations of the sample stage, both systems allow micro-beam scanning and thus lithography possible. The 
beam shutter to start or stop the beam is also controlled by a computerized rotation motor. Two beam fluence monitoring methods are used. The first approach uses a rotating dual vane which is coated with gold, as shown in Fig. 2a, for in-situ beam fluence monitoring. This is based on a measurement of the backscattered ion yield, determined by the Rutherford Backscattering Spectrometry (RBS), in combination with synchronous current measurement. In the setup, the Au-coated dual vane rotator, of $30^{\circ}$ angular span for each vane in $5 \mathrm{~cm}$ length with a total mass of $3.82 \mathrm{~g}$, rotates with a constant angular velocity of $6.15 \mathrm{rad} / \mathrm{sec}$ by a unipolar stepper motor to periodically chop the beam. A SSB detector connected to a pre-amplifier, an amplifier, a multichannel analyzer (MCA) and a PC in series is used to collect the number of backscattered ions at $150^{\circ}$ when the beam is blocked by the vane. The beam impinges at about $4 \mathrm{~cm}$ from the rotating center of the vane. This allows $83.3 \%$ of the beam passing through the vane [12]. The second approach is simply based on the measurement of the ion charge intercepted by the Lshaped aperture blades during the beam exposure. The input charge ratio between onto the blades and on the exposed sample area, which should be in principle proportional to the ratio between the two areas, is predetermined by calibration. Then, in the experiment the charge input by the beam onto the aperture blades transformed to the charge on the sample [10].

The tapered glass microcapillary system, as shown in Fig. 3, consists of a 0.8 -mm beam collimator to guide the beam into the capillary tube, the glass tapered capillary tube fixed in an $\mathrm{Al}$ frame, and a rotator which is connected by a carbon fiber rod with the $\mathrm{Al}$ frame to move the glass tube away or into the beam direction. The sample holder is $\mathrm{X}-\mathrm{Y}$ moveable with a translation repeatability of $4 \mu \mathrm{m}$ and an accuracy of $6 \mu \mathrm{m}$. The electronic and software drivers of the system are in-house developed including the slider backlash compensate. In the front of the holder a $120-\mu \mathrm{m}$ pinhole slit is installed to limit the final beam size for routine irradiation 
experiments. A Faraday cup is placed beside the sample on the holder to monitor the beam current. Various sized glass capillary tubes (as shown in Fig. 4) can be in-house fabricated using a home-developed glass microcapillary puller based on the induction heating technique [13]. The glass capillary in different sizes is changeable to install in the capillary holder. After each time of the installation, beam alignment is firstly carried out with the help of using a laser beam. A laser source is installed under the beam line just in the front of the endstation and the laser beam is deflected by a rotatable mirror inside the beam line to direct to the capillary (the laser beam was originally well aligned with the ion beam). Simple manual adjustment of the capillary can finally achieve a satisfying alignment by looking at the best laser beam spot at the target position through the capillary (certainly this is a time-consuming process). The microbeam quality at the target as a function of the distance between the capillary exit and the target was studied. As seen in Fig. 5 for example, the quality of the beam spot is generally improved when the beam core homogeneity increases as increasing of the distance up to about $2 \mathrm{~cm}$, while the halo region increases too, indicating the ion fluence decreasing in the core region and thus less PMMA modification. Based on the studying result, in irradiation experiments the distance between the capillary exit and the sample surface is normally set not greater than $20 \mathrm{~mm}$ depending on the capillary size.

\section{Applications}

Both programmed L-shaped blade aperture and tapered glass capillary systems were applied to $\mathrm{MeV}$ proton microbeam lithography. For operation, installation of the two systems was interchangeable within a four-column frame inside the endstation chamber (Fig. 2b and Fig. 6). Normally the capillary microbeam had a relatively higher beam current or intensity due to a 
focusing effect compared with the aperture microbeam (e.g. at the same beam size, such as 100 $\mu \mathrm{m}$, the former had a current of several $\mathrm{nA}$ whereas the latter had about $1 \mathrm{nA}$ for $2 \mathrm{MeV}$ proton beam), while the latter had a more homogeneous distribution of the beam intensity in the beam spot than the former which had a halo effect. Therefore, for high-quality lithography the aperture microbeam was applied, while for fast but not very high quality lithography the capillary microbeam was applied. The materials irradiated included either positive tone or negative tone poly(methyl methacrylate) (PMMA), poly(dimethylsiloxane) (PDMS) and amorphous silica $\left(\mathrm{SiO}_{2}\right)$. Exposure characteristics of the materials to the microbeam were studied for fluence conditions satisfying production of good quality microstructures. In order to produce microstructures in "positive" resist tone of PMMA, the ion fluence $\Phi$ should satisfy the condition of $\Phi_{0} \leq \Phi<\Phi_{\mathrm{x} 0}$, where $\Phi_{0}$ and $\Phi_{\mathrm{x} 0}$ are the cleaning and onset of cross-linking ion fluences, respectively, whereas for producing "negative" tone resist the fluence should be $\Phi_{\mathrm{x} \infty} \leq$ $\Phi$, where $\Phi_{\mathrm{x} \infty}$ is the full cross-linking ion fluence. $\Phi_{0}, \Phi_{\mathrm{x} 0}$ and $\Phi_{\mathrm{x} \infty}$ were measured for various ion beam conditions [14] so that an applied ion fluence for a certain material could be determined from the database. In using apertures for microbeams, concerns are normally on the beam scattering caused divergence behind the aperture. However, our experimental results showed that in the utilization of the L-shaped aperture for microbeam, the aperture edge scattering did not significantly affect the pattern edge sharpness $[3,15]$ so that the pattern quality could be guaranteed. Some examples of applications in $\mathrm{MeV}$ microbeam lithography using the L-shaped micro-aperture have been reported [10]. In a recent investigation on blister formation on PMMA induced by the aperture microbeam, we determined the blister-free condition of 2$\mathrm{MeV}$ proton beam which should be with a flux less than $4.7 \times 10^{11}$ ions $/ \mathrm{cm}^{2} \mathrm{~s}$. In the tapered glass micro-capillary writing of micro-patterns, a pattern generation system consisting of two 
parts has been developed. The first part is a stand-alone pattern design program that operates similarly to a paint program. The drawing pad has a selectable scale of $1000 \times 1000$ or $10000 \times 10000 \mu \mathrm{m}^{2}$ with a resolution of $1 \mu \mathrm{m}$. The drawing tools consist of a line tool, a freehand drawing tool and a point tool with a selectable point size which is the beam spot size. The second part is the pattern writer, which is integrated into the $x / y$ target stage motor control software. An $\mathrm{x} / \mathrm{y}$ data pair is read into the program from a file, the exposure time between drawing points is set, and the pattern is automatically written to the target. Fig. 7 shows an example of patterns written in such way and the sharpness of the written pattern edge. The focusing factor of the tapered glass capillary was measured to be $f=2.7$, which was fairly high. This is an advantage of the tapered capillary for microbeam lithography over the aperture chopped microbeam due to the focusing effect and thus higher beam intensity to lead to shorter processing time. In a recent new development, the applications of the tapered glass microcapillary system are being extended to $\mathrm{MeV}$ microbeam PIXE mapping/imaging (Fig. 8 shows an example). This extension is simple with placing the target sample on the sample stage and $x-y$ translating the stage step by step for the capillary-focused microbeam to perform PIXE analysis point by point on the sample surface.

\section{Conclusion}

Economic systems of the programmable L-shape micro-aperture and the tapered glass micro-capillary have been developed for $\mathrm{MeV}$ microbeams at Chiang Mai University. The aperture system is capable of varying the aperture size down to $1 \mu \mathrm{m}$ in minimum by full computer controls of beam fluence monitoring and pattern writing. The capillary system is equipped with an in-house-made tapered glass capillary with a $\mu$ m-exit. Cooperating with X-Y translations of the sample stage, both systems allow micro-beam scanning and thus $\mathrm{MeV}$ ion 
beam lithography possible. The materials irradiated included either positive tone or negative tone poly(methyl methacrylate) (PMMA), poly(dimethylsiloxane) (PDMS) and amorphous silica $\left(\mathrm{SiO}_{2}\right)$. Exposure characteristics of the materials to the microbeam were studied for fluence conditions satisfying production of good quality microstructures. Experimental results showed that the aperture edge scattering did not significantly affect the pattern edge sharpness so that the pattern quality could be guaranteed. PMMA transformation was observed such that at the threshold fluence of $3.5 \times 10^{14}$ ions $/ \mathrm{cm}^{2}$ for $2-\mathrm{MeV}$ proton beam the polymer transformed from a positive to a negative resist. Applications were focused on fabrication of microfluidic device patterns and further extended to mapping of biological samples.

\section{Acknowledgements}

The work has been supported by the Royal Golden Jubilee (RGJ) Scholarship of the Thailand Research Fund (TRF), the Ph.D. Scholarship of the Thailand Center of Excellence in Physics, the Development and Promotion of Science and Technology Talents Project, Thailand, the Academy of Finland Centre of Excellence in Nuclear and Accelerator-Based Physics (Ref. 213503), and the International Atomic Energy Agency (IAEA). We wish to thank Chome Thongleurm for the technical assistance in the accelerator and Michael W. Rhodes for the technical assistance in electronics.

\section{References}

[1] B.D. Michael, M. Folkard and K.M. Prise. Meeting Report: Microbeam Probes of Cellular Radiation Response, 4th L.H. Gray Workshop, 8-10 July 1993. Int. J. Radiat. Biol. 65 (1994) 503-508. 
[2] International Atomic Energy Agency (IAEA), Database of Electrostatic Accelerators, Accelerator Knowledge Portal, https://nucleus.iaea.org/sites/accelerators/Pages/ADB.aspx. 2016.

[3] N. Puttaraksa, S. Gorelick, T. Sajavaara, M. Laitinen, S. Singkarat, H.J. Whitlow, Programmable proximity aperture lithography with $\mathrm{MeV}$ ion beams, J. Vac. Sci. Tech. B 26(5) (2008) 1732-1739.

[4] F. Watt, M.B.H. Breese, A.A. Bettiol, J.A. van Kan, Proton beam writing, Mater. Today 10(6) (2007) 20-29.

[5] S. Sangyuenyongpipat, H.J. Whitlow, S.T. Nakagawa, E. Yoshida, Lithography with MeV Energy Ions for Biomedical Applications: Accelerator Considerations, AIP Conf. Proc. 1099(1) (2009) 282-286.

[6] T. Nebiki, T. Yamamoto, T. Narusawa, M. B. H. Breese, E.J. Teo and F. Watt, Focusing of MeV ion beams by means of tapered glass capillary optics, J. Vac. Sci. Technol. A 21 (2003) 1671-1674.

[7] T. Nebiki and T. Narusawa, Glass capillary optics for focusing of high energy ion beams, International Symposium on Discharge and Electrical Insulation in Vacuum 2006, ISDEV'06, International Symposium $\quad$ Matsue, 2 (2006) 813-816. (DOI: 10.1109/DEIV.2006.357427)

[8] T. Kobayashi, T.S. Miyamoto, T. Ikeda, T.M. Kojima, K. Ogiwara, Y. Yamazaki, Surface modification of polymers by ion irradiation at the solid-liquid interface, Nucl. Instr. Meth. B 272 (2012) 405-408. 
[9] T. Ikeda, Y. Kanai, T.M. Kojima, Y. Iwai, T. Kambara, Y. Yamazaki, M. Hoshino, T. Nebiki, T. Narusawa, Production of a microbeam of slow highly charged ions with a tapered glass capillary, Appl. Phys. Lett. 89(16) (2006) 163502-3.

[10] N. Puttaraksa, S. Unai, M.W. Rhodes, K. Singkarat, H.J. Whitlow and S. Singkarat, Fabrication of a negative PMMA master mold for soft-lithography by $\mathrm{MeV}$ ion beam lithography, Nucl. Instr. Meth. B 272 (2012) 149-152.

[11] Somrit Unai, Focusing and Flux/Fluence optimization of $2 \mathrm{MeV}$ Proton Microbeam for Ion Beam Lithography Applications, Ph.D. thesis, Chiang Mai University, April 2013.

[12] N. Puttaraksa, M.W. Rhodes, T. Kamwanna, U. Tippawan, C. Thongleurm, W. Ginamoon, H.J. Whitlow, S. Singkarat, In-situ ion beam fluence monitoring system for $\mathrm{MeV}$ ion beam lithography at CMU, Thai Journal of Physics, Series 5 (2010) 163.

[13] S. Unai, M.W. Rhodes, C. Sriprom, K. Singkarat, N. Pussadee, S. Singkarat, A tapered glass microcapillary processing system for focusing a $\mathrm{MeV} \mathrm{H}^{+}$ion beam, Chiang Mai Journal of Science 40(4) (2013) 789-797.

[14] N. Puttaraksa, R. Norarat, M. Laitinen, T. Sajavaara, S. Singkarat, H.J. Whitlow, Lithography exposure characteristics of poly(methyl methacrylate) (PMMA) for carbon, helium and hydrogen ions, Nucl. Instr. Meth. B 272 (2012) 162-164.

[15] S. Gorelick, T. Sajavaara, M. Laitinen, N. Puttaraksa, and H.J. Whitlow, Resolution performance of programmable proximity aperture $\mathrm{MeV}$ ion beam lithography system, MRS Proceedings V 1020 (2007) 1020-GG03-04. 


\section{Figure Captions:}

Figure 1. Schematic diagram of the beam line of the 1.7-MV Tandetron accelerator and beam line at Chiang Mai University. Both microbeam systems are alternatively installed inside the chamber which is normally used for ion beam analysis. The beam line length is about $10 \mathrm{~m}$.

Figure 2. Programmable L-shape blade micro-aperture system. (a) Schematic of the in-situ beam fluence monitoring system. (b) A photograph of the aperture positioning system with a close-up view at the right.

Figure 3. Tapered glass micro-capillary beam focusing system. (a) Schematic diagram of the system. In the shown case, an SSD detector with a 300- $\mu$ m slit at the front of it is used at the target position for ion energy distribution measurement, while in normal irradiation experiments, a $120-\mu \mathrm{m}$ slit is used in the front of target sample. (b) A photograph of the system. (c) and (d) Close-ups of the capillary holder.

Figure 4. Home-fabricated tapered glass micro-capillaries. (a) A photo of tapered glass microcapillaries which were pulled by different weights of 1, 3, 5, 7 and $9 \mathrm{~N}$ (from bottom to top). (b) A cross-section image of the capillary pulled by a 9-N weight. (c) Side view of the tip part of a microcapillary pulled by double stretching method. 
Figure 5. An example of the study on the capillary microbeam spot as a function of the distance between the capillary exit and the sample surface. In this experiment, the ion beam was of 2$\mathrm{MeV}$ protons, the capillary exit inner diameter was $500 \mu \mathrm{m}$, and the sample material was PMMA film on Si wafer.

Figure 6. A top-view photograph of the four-column frame inside the endstation chamber to support and position the microbeam systems. The photo currently shows the microcapillary system installed in the frame.

Figure 7. Demonstration of microcapillary in proton microbeam lithography. (a) A photo of the tapered glass microcapillary used. (b) An original pattern designed by freehand writing via home-developed drawing software. (c) An optical microscope image of the developed pattern which was transferred from (b) onto PMMA film by proton beam writing through the capillary in (a). (d) A close-up zoomed from a corner of the written letter to show the sharpness of the pattern edge. The irradiating beam condition: $2-\mathrm{MeV}$ proton beam to $1.3 \times 10^{14}$ ions $/ \mathrm{cm}^{2}$.

Figure 8. $\mathrm{MeV}$ proton microbeam mapping of the vein edge part of a local longan leaf. (a) A microphotograph of the analyzed leaf part. The white box indicates the PIXE mapping area. The leaf vein is seen about $800 \mu \mathrm{m}$ in width. (b) Elemental maps (in 1D) of the vein-leaf part. The concentrations (in \%) are normalized for all of the detected elements. 


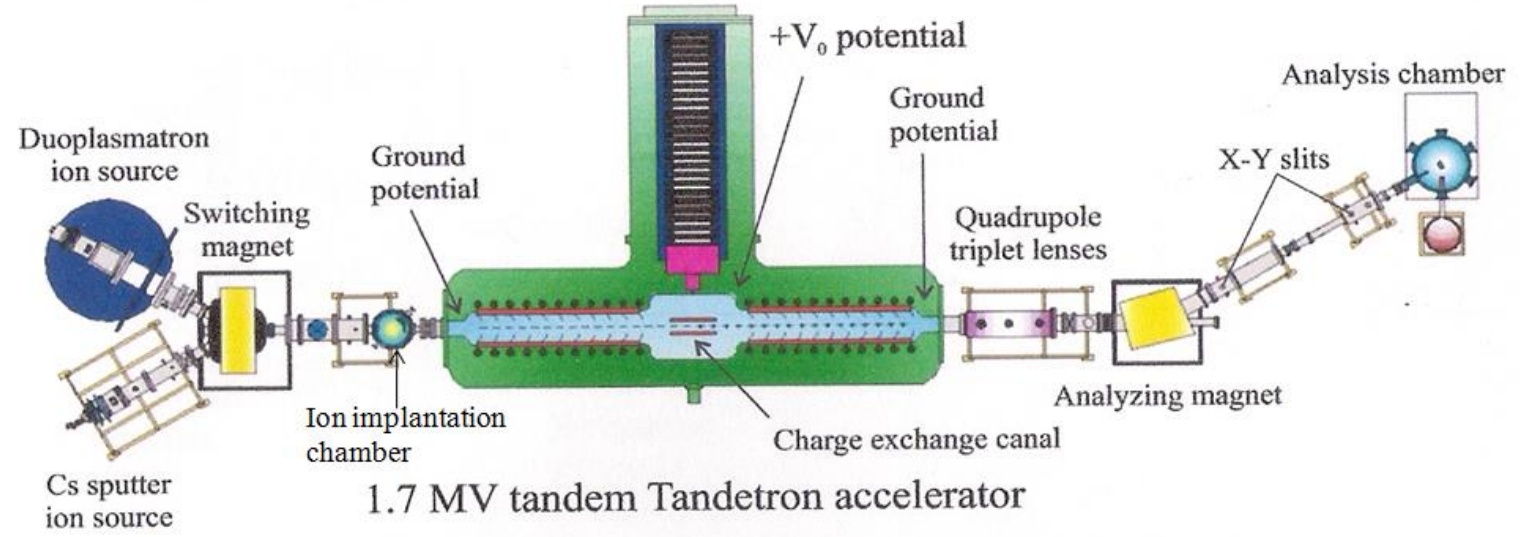

Figure 1 

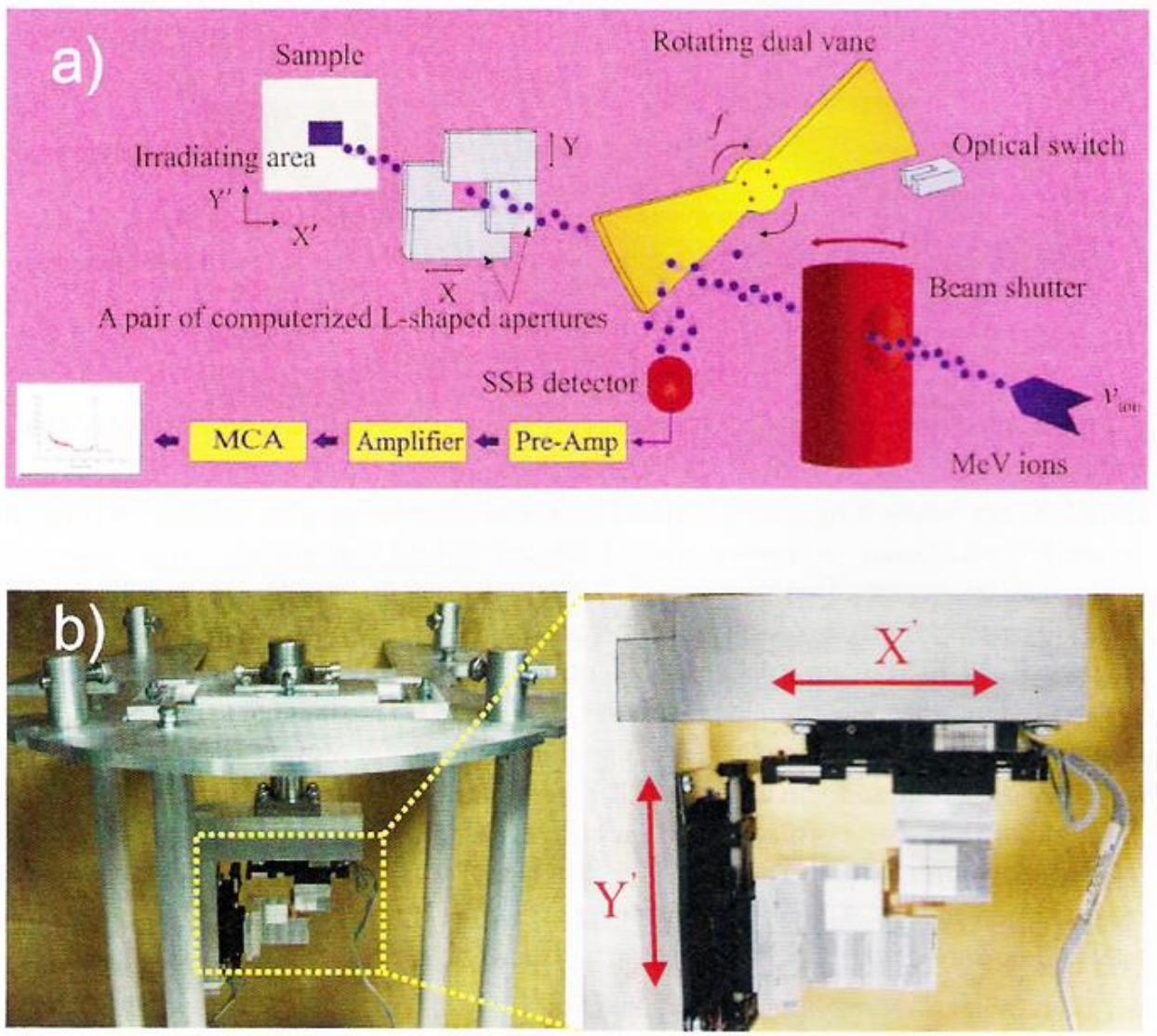

Figure 2 

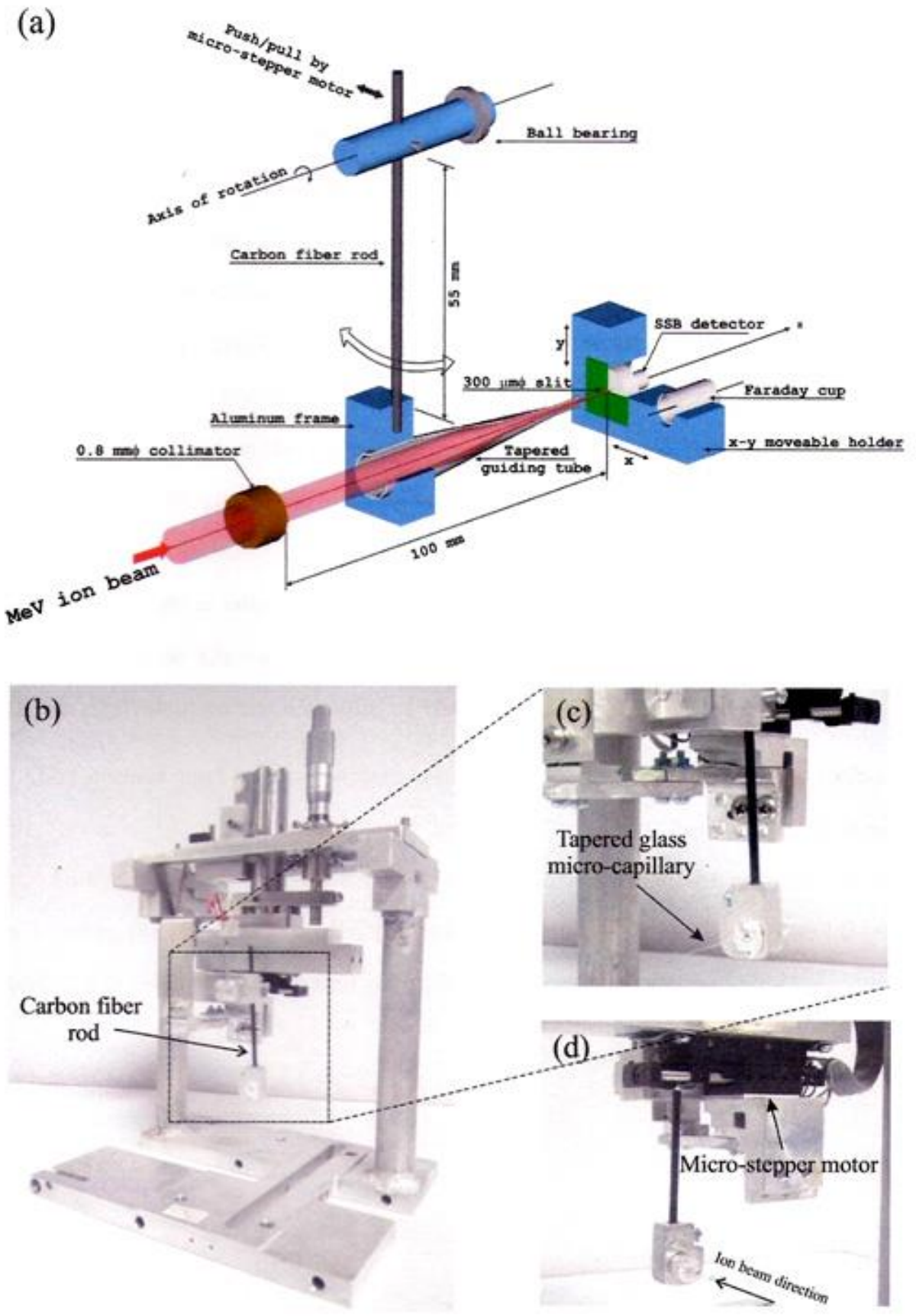

Figure 3 

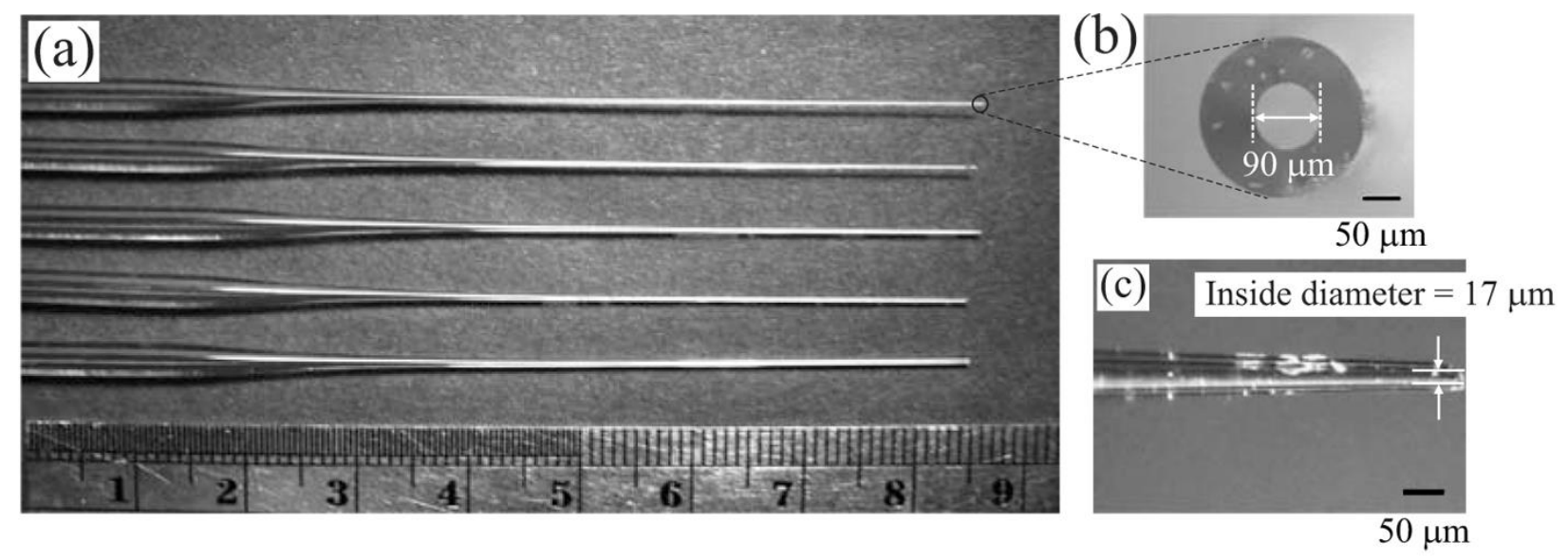

Figure 4

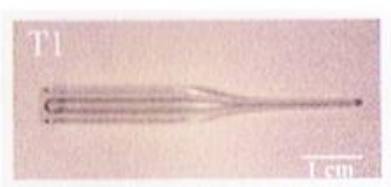

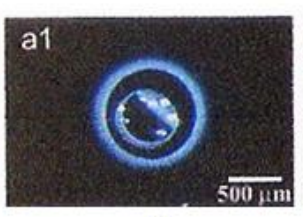

5

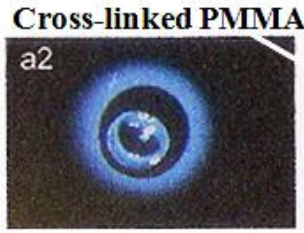

10

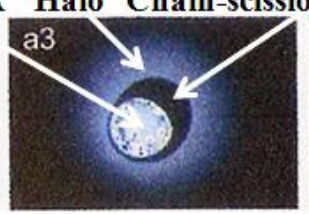

14

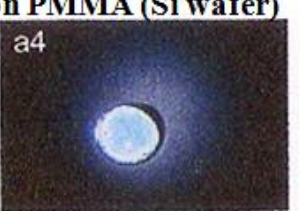

22

Distance between capillary exit and sample surface $(\mathrm{mm})$

Figure 5 


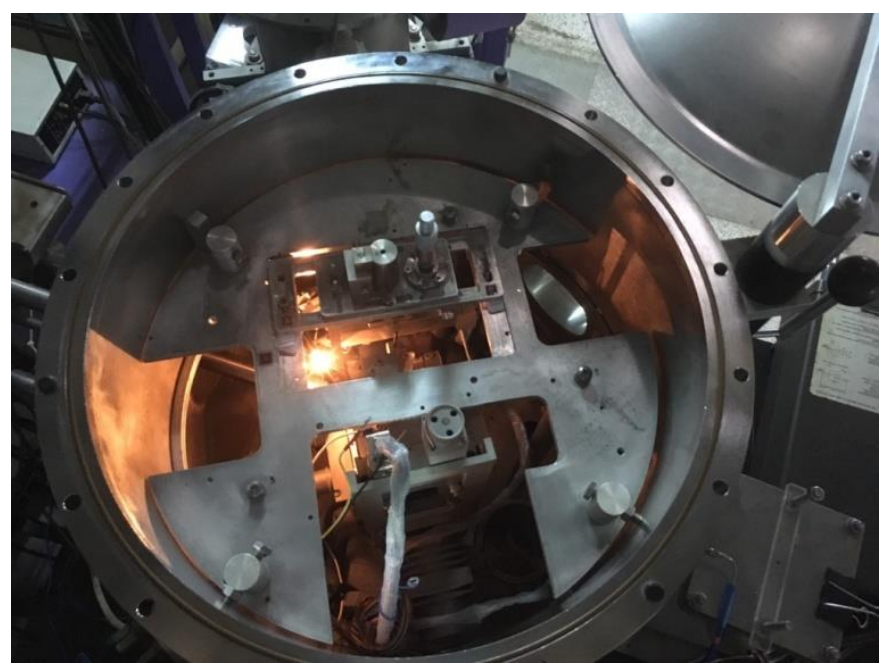

Figure 6

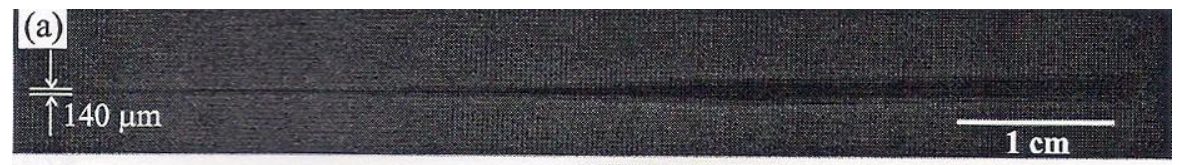

(b)

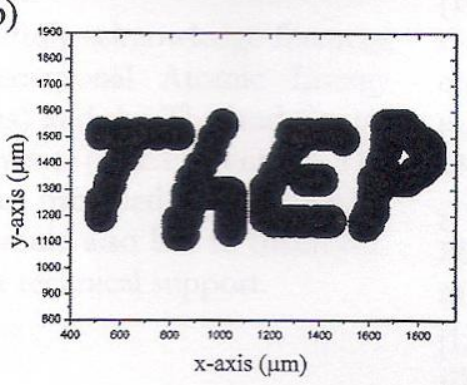

(c)

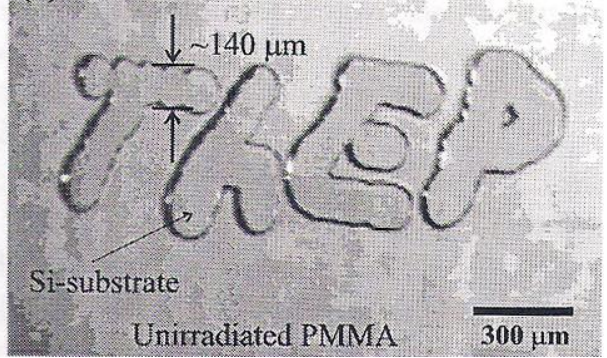

(d)

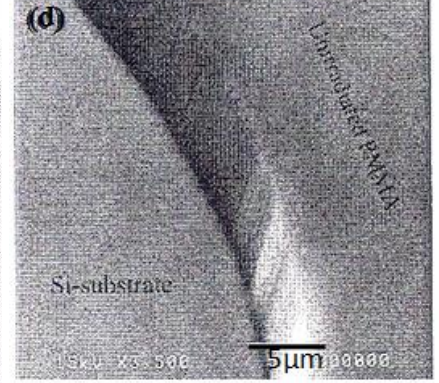

Figure 7 


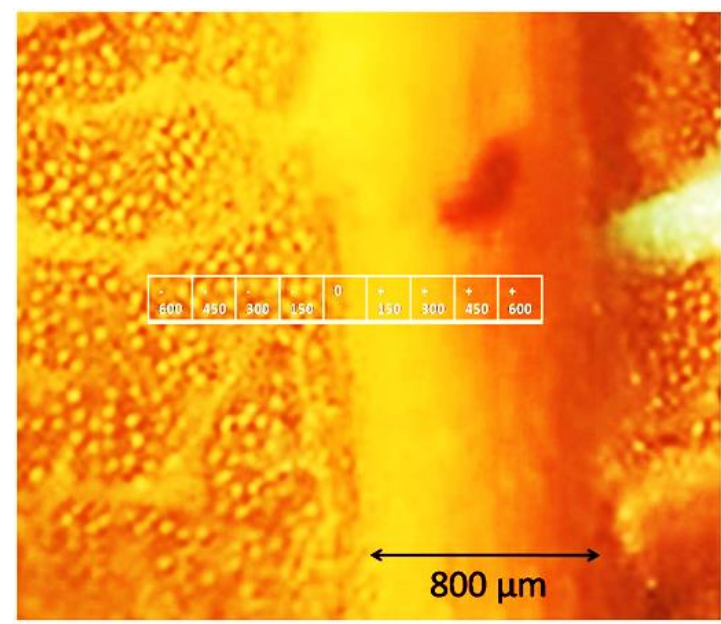

(a)

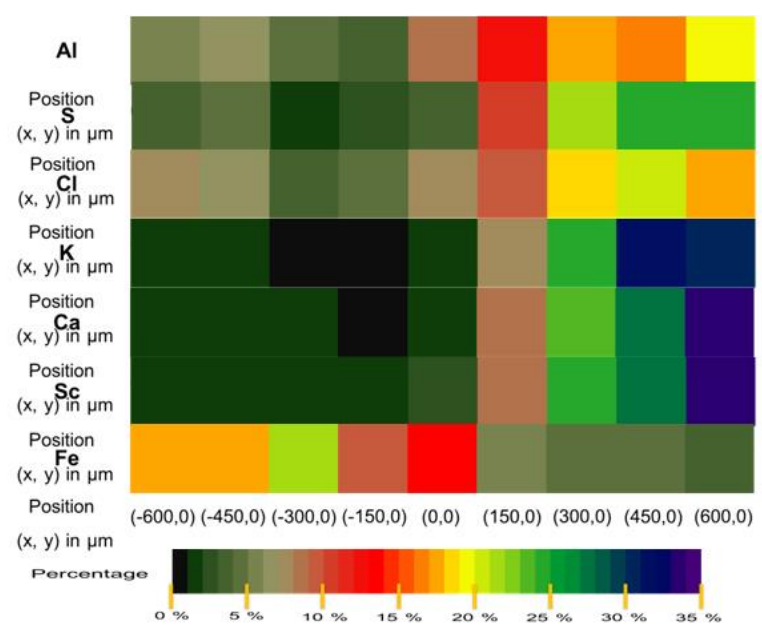

(b)

Figure 8 